\title{
THE IMPACT OF PLATFORM SCREEN DOORS ON RAIL CAPACITY
}

\author{
O. LINDFELDT \\ Sweco AB, Stockholm, Sweden.
}

\begin{abstract}
A new railway line for commuter trains through central Stockholm, the 'City line', is under construction and will open in 2017. The line will have two new underground stations and these will be equipped with platform screen doors in order to enhance air quality and prevent suicides. Several stations along the line will have only two platform tracks and this will limit capacity.

Neither trains nor platform doors will be fully automatized and thus the drivers will have to stop the trains in exact position to match the platform doors. Consequently, adding extended times for opening and closing doors will prolong the dwell time by approximately $12 \mathrm{~s} / \mathrm{stop}$.

An extensive capacity evaluation has been carried out as a RailSys simulation in order to find out whether a capacity of 24 trains/hour/ direction can be reached. The core part of the study was to prepare dwell time distributions for all stations along the line. These distributions include several terms, namely additional braking time due to a more careful and precise braking, time to correct stopping position (stochastic), door opening time, passenger exchange time (stochastic), door closing time, door obstruction time (stochastic) and driver's reaction time before departure.

The simulation results indicate that the extended dwell times caused by the platform screen doors limit capacity to 22 trains/hour in one direction and 24 trains/hour in the other direction. This difference comes from the fact that the station with the longest dwell times is located adjacent to a station with four platform tracks where trains can recover delays. Thus, the delay level differs significantly between the two traffic directions. This delay difference influences the useful capacity in such a way that the most punctual direction has a higher capacity.

Keywords: dwell time, platform screen doors, railway capacity, simulation.
\end{abstract}

\section{INTRODUCTION}

A new railway line through central Stockholm, called 'City line', is under construction and will open in 2017. The line will be used as a commuter line. As a result the commuter traffic through central Stockholm will be completely separated from the regional and long-distance traffic. The traffic on the City line will be completely homogenous.

The line has two new underground stations, which are considered to be very attractive and frequently used by commuters in the future. These stations will be equipped with platform screen doors in order to enhance air quality and prevent suicides.

The Stockholm commuter network is X-shaped and consists of two branches to the north and two branches to the south (Fig.1). The City line will be a common, connecting link in the middle of the network. The overreaching goal is to operate all four branches with a regular timetable with five minutes between trains, which gives 24 trains/hour/direction through the City line.

However, since the train operation is not to be automatized, all trains will still be driven by human drivers. Moreover, the platform screen doors cannot be fully adjusted to the commuter

\footnotetext{
This paper is part of the proceedings of the 15th International Conference on Railway Engineering Design and Operation (COMPRAIL)

CONFERENCES www.witconferences.com
} 


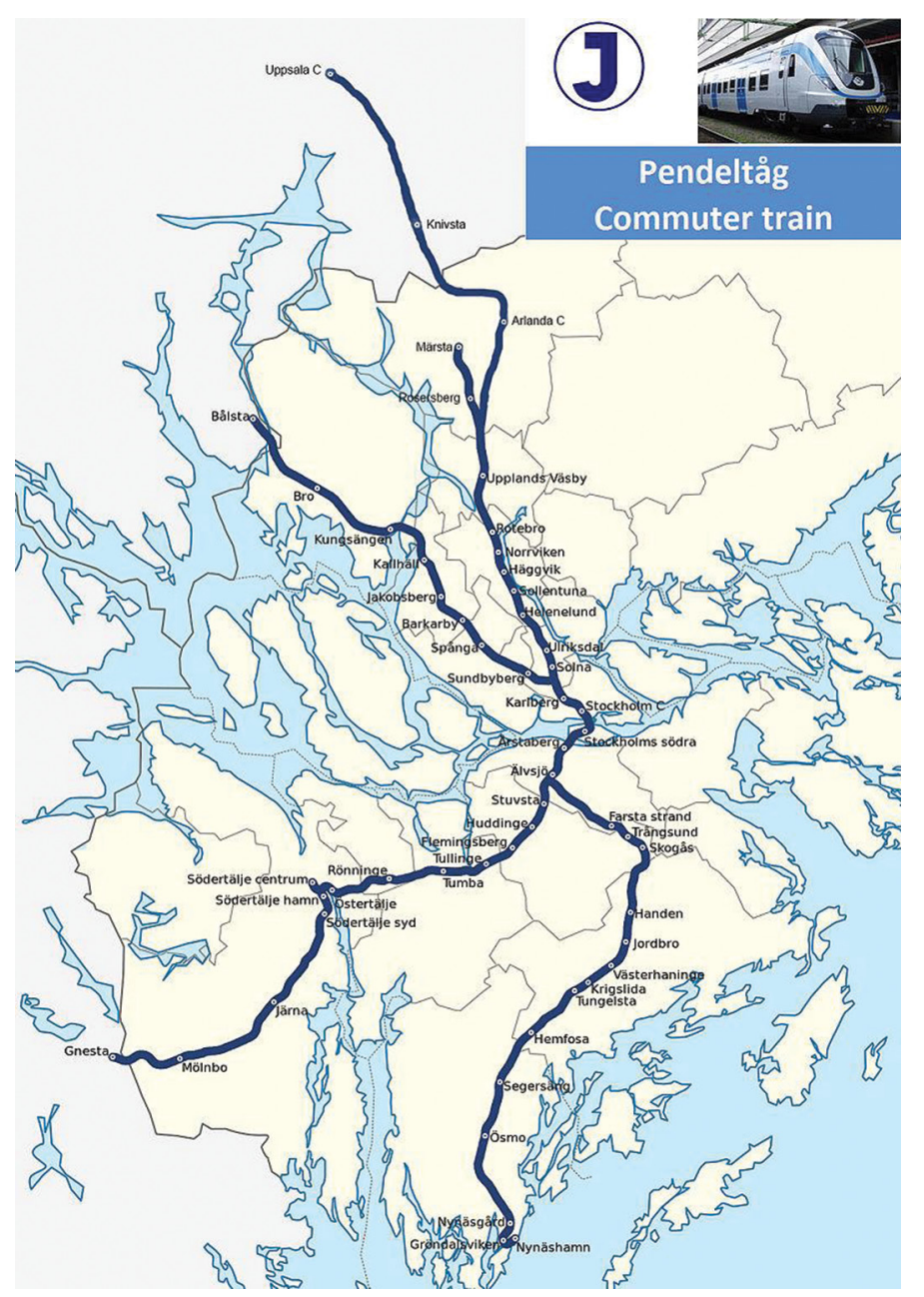

Figure 1: Stockholm's commuter train network.

train vehicles, since the City line ought to be used also as redundancy for other train categories. This implies that the screen doors will prolong the dwell time and affect capacity negatively.

In order to find out whether the capacity of 24 trains/hour/direction can be reached, an extensive capacity evaluation has been carried out as a RailSys simulation.

A crucial part of the work was to prepare distributions for the future dwell times at the stations. To do this, a special model for the dwell times was developed. Important parts of the model were extended braking time due to careful and precise stopping, door opening times, passenger alighting and boarding times, door closing times, extra dwell time caused by door obstructions, and driver's reaction time before departure. Several of these parts are stochastic and probability methods were applied in order to combine several stochastic contributions to the overall dwell time distributions derived. These distributions were used as input in the simulation.

Simulations were performed for different levels of traffic load, i.e. number of trains per hour. Results were compiled for mean delay and punctuality and from these a level of useful capacity could be found for each traffic direction.

This paper describes the procedure used to compile the dwell time distributions, the successive simulation as well as the final analysis, results and capacity recommendations. 


\section{LIMITATIONS}

The study is a detailed simulation study of the mid-section of the commuter system in Stockholm. It is delimited in the following three important ways:

- geographically

- through delay modelling

- in time.

It is suitable to delimit the analysis to the section between the junctions where the two lines merge north and south of Stockholm. In order to make the simulation of the merging flows in the junctions realistic, the studied area also included the first station beyond each junction.

The simulated system is shown schematically in Fig. 2. Northbound trains are initiated in Älvsjö, whereas southbound trains are initiated in Sundbyberg and Solna. This makes it possible to model the critical merging procedures north of Älvsjö and south of Sundbyberg/ Solna. The rest of the network is modelled indirectly through the use of distributions for the initial delays in Sundbyberg, Solna and Älvsjö, as will be discussed later in this paper.

Disturbances will also cause delays to trains within the simulated area. These can be divided into two categories: dwell time extensions and run-time extensions between scheduled stops. The former are in focus in this study, whereas the latter are not taken into account. This means that the effect of infrastructure, vehicle malfunctions, etc., is not further analysed and modelled.

The system will be fully utilized twice a day: during the morning peak period and during the afternoon peak period. For this reason only a time period of three hours, between 06:00 a.m. and 09:00 a.m., is evaluated in the simulation. This peak period is preceded and followed by a period with off-peak traffic in order to warm up the simulation model. Only trains operated during the peak traffic period are evaluated.

Figure 2 shows that the line has four stations between the junctions. Three of these stations have only two platform tracks, whereas the Stockholm City station has four tracks. Due to this configuration, the two-track stations will limit capacity and the dwell time in these stations will be critical for overall line capacity.

Platform screen doors will be installed in two underground stations: Stockholm City and Stockholm Odenplan. These doors require the trains to stop in a specific position in order to get a match between the train doors and the doors on the platform. Three alternative procedures for door closing and departure procedure are considered.

1. Platform screen doors are closed separately by the conductor.

2. Platform screen doors are closed automatically when train doors are closing. The conductor monitors the closing procedure while standing on the platform.

3. It is similar to alternative 2 , but the conductor monitors the closing procedure while standing in his cabin on board of the train.

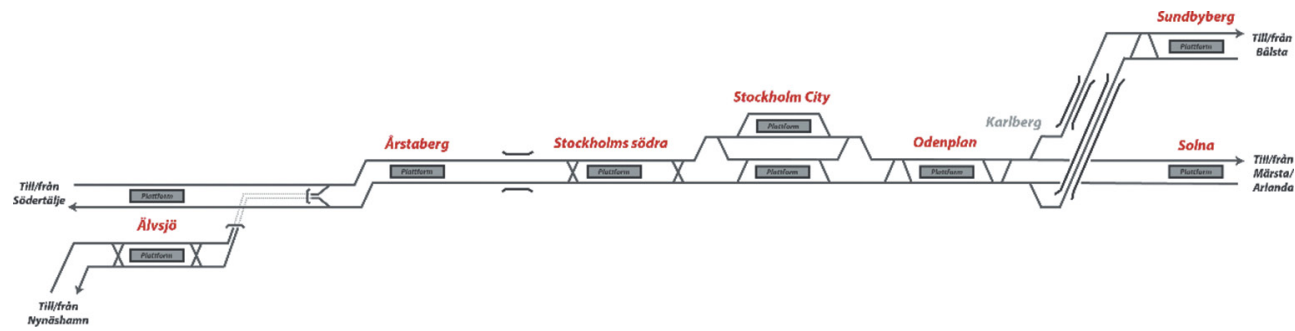

Figure 2: The analysed line section. 
The three closing procedures are different in the sense that the total time - from initiation of the door closing procedure until clearance to drive is announced to the driver by the conductor - is estimated to be 16,17 and $15 \mathrm{~s}$, respectively.

\section{TRAFFIC, TIMETABLES AND INITIAL DELAYS}

A micro-simulation analysis of railway traffic requires a timetable to be designed and simulated (see Lindfeldt [1] and Sipilä [2]). Four alternative timetables, representing different capacity utilization levels, have been designed and simulated in order to find out how the platform screen doors impact the overall line capacity. Since traffic is completely homogenous it is easy to spread the trains evenly over the hour. The analysed timetables contained 20, 22, 24 and 26 trains/hour/direction. These traffic loads correspond to a time separation of 180 , 164, 150 and $138 \mathrm{~s}$ between adjacent trains, respectively.

A fifth timetable, with only 2 trains/hour/direction, was evaluated as a reference. At this low-traffic density the knock-on delays are negligible, due to the large time separation, which means that the results will only contain primary delays and the robustness will be maximal.

All timetables were constructed so that every second train arrives from each of the two connecting branches in each junction.

Two important types of delay were modelled: initial delays and dwell time extensions. These are important inputs to the simulation model and have to be derived and compiled carefully (see Pachl [3] and Yuan and Hansen [4]).

Initial delays were assigned to the trains in Älvsjö, Sundbyberg and Solna through distributions that were compiled from historical delay data (Swedish Transport Administration [5]). Altogether four different distributions were compiled from the data for approximately 200 days of operation (Fig. 3).

These delays reflect the impact of the surrounding area, and they result in stochastic conflict situations that consume capacity in the City line. The cost, in the form of added delay, will determine the useful capacity, i.e. the number of trains that can be scheduled through the line.

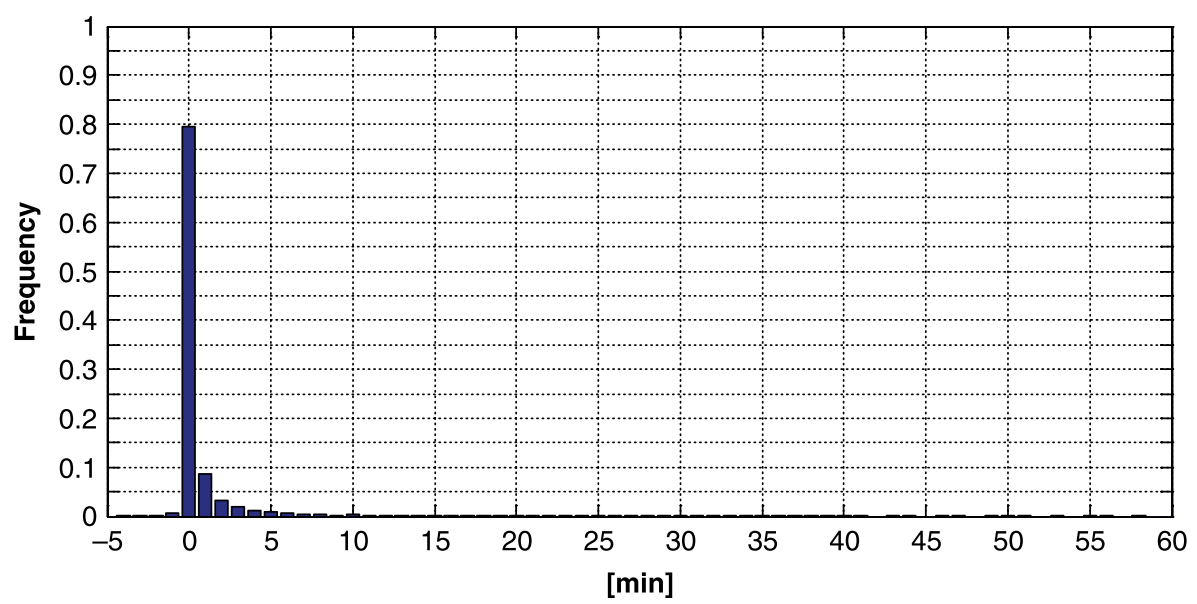

Figure 3: Initial delay distribution. This example is for trains from Älvsjö, bound for Solna. 


\section{DWELL TIME MODELLING}

The dwell times are core inputs in the analysis, since they are directly affecting the capacity. A strong correlation is expected between capacity and the existence of platform screen doors, since these doors will affect dwell times and hereby also capacity.

Each station and traffic direction will have an individual distribution for the dwell time and it is essential to estimate these correctly to get reliable results from the simulation analysis (Yuan and Hansen [4]). The modelled area has four stations where delay distributions are needed as input in the simulation model:

\section{- Årstaberg and Stockholms Södra}

These are existing stations that will not (initially) be equipped with platform screen doors. The number of passengers using these stations is not expected to increase significantly compared to the current situation.

- Stockholm City and Stockholm Odenplan

These are newly constructed underground stations and the number of passengers is expected to increase, compared to the corresponding existing stations. Platform screen doors will be installed in these stations.

Dwell time distributions for the two existing stations, Årstaberg and Stockholms Södra, are rather easy to obtain through field time measurements. Such measurements were carried out on the platforms, analysed and compiled to one distribution, since the differences between the two stations and the two directions were small.

Figure 4 shows the distribution. The standard deviation is rather low and a scheduled dwell time of $45 \mathrm{~s}$ would be enough to cover most train stops without increase in delay. The mean dwell time is $38 \mathrm{~s}$.

Finding the corresponding dwell time distributions for the two stations that will be equipped with platform screen doors is not this straightforward. These stations are non-existing and the size of the passenger flows is unknown. These flows affect the time for passenger exchange,

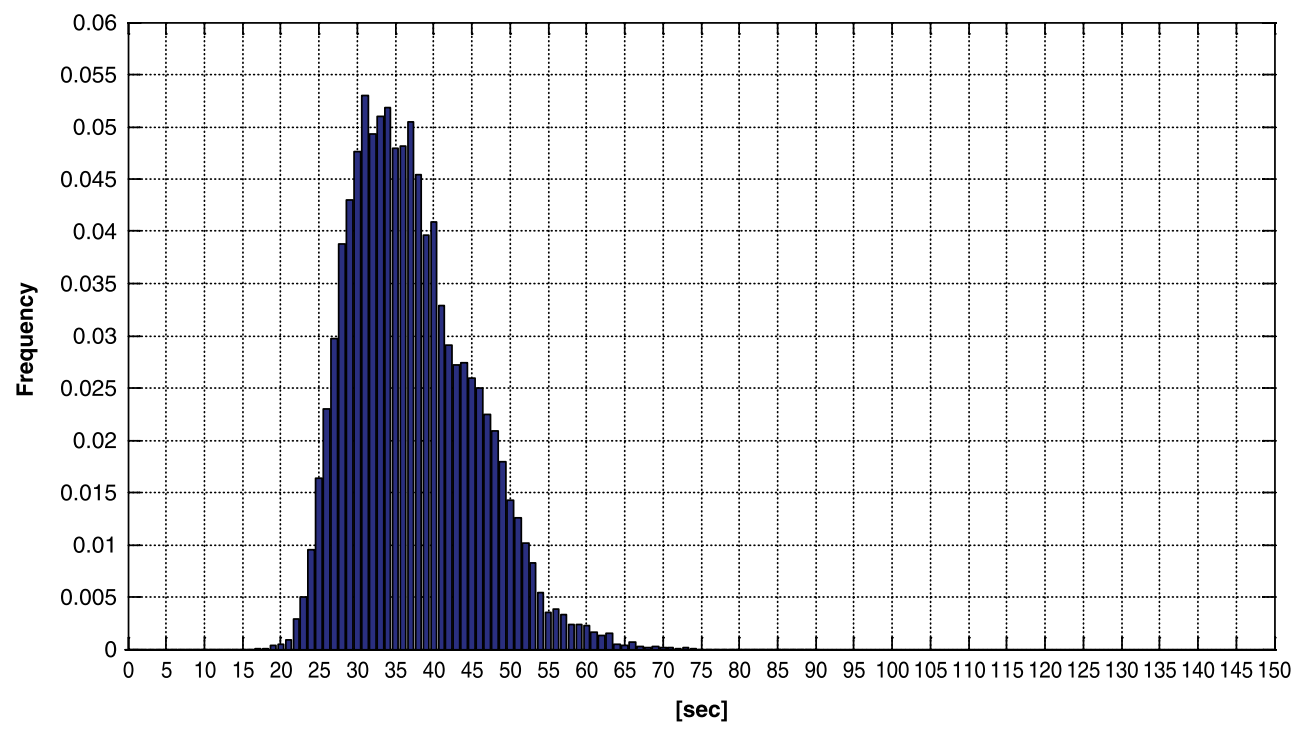

Figure 4: Dwell time distribution for Årstaberg and Stockholms Södra. 
i.e. alighting and boarding. Moreover, the platform screen doors will affect the dwell time in several ways.

A first assumption, which might have to be corrected in a later study, is to make use of measured time distributions for alighting/boarding at existing stations. Assuming nonaffected times for passenger exchange would imply that the City line will not increase the number of passengers. This assumption might hold as long as the increased passenger numbers are compensated with additional trains, i.e. as long as the number of alighting/boarding passengers per train is approximately the same as in the existing system.

A special model was set up in order to include the effect of the platform screen doors on the dwell time distributions (see Fig. 5). The model contains the following seven terms, correlated to the arrival, dwell or departure of a train:

- additional braking time

- time to correct stopping position (reverse the train a few metres)

- door opening time

- passenger exchange time

- door closing time

- door obstruction time

- driver's reaction time.

The platform screen doors impose the drivers to brake more carefully in order to stop the train within a stopping zone of approximately $0.5 \mathrm{~m}$. This braking phase will take place on the platform track circuit, which means that the additional time consumed by the more carful braking will increase occupation time of the block section that is critical for capacity. This factor is assumed to be a constant that is equal to 4 s/arriving train.

From time to time, drivers will miss the stopping zone and have to reverse the train a couple of metres. Such a reverse procedure has been measured to take about $14 \mathrm{~s}$. The occurrence of this situation is estimated to be $1 / 30$. The stochastic feature of this factor is marked as dashed in the figure.

Door opening time has been measured to be $6.5 \mathrm{~s}$. This time includes a short delay, compared to a situation without any platform screen doors.

The passenger exchange time depends only on the number of alighting and boarding passengers and their distribution on the train doors. As discussed earlier, these time distributions

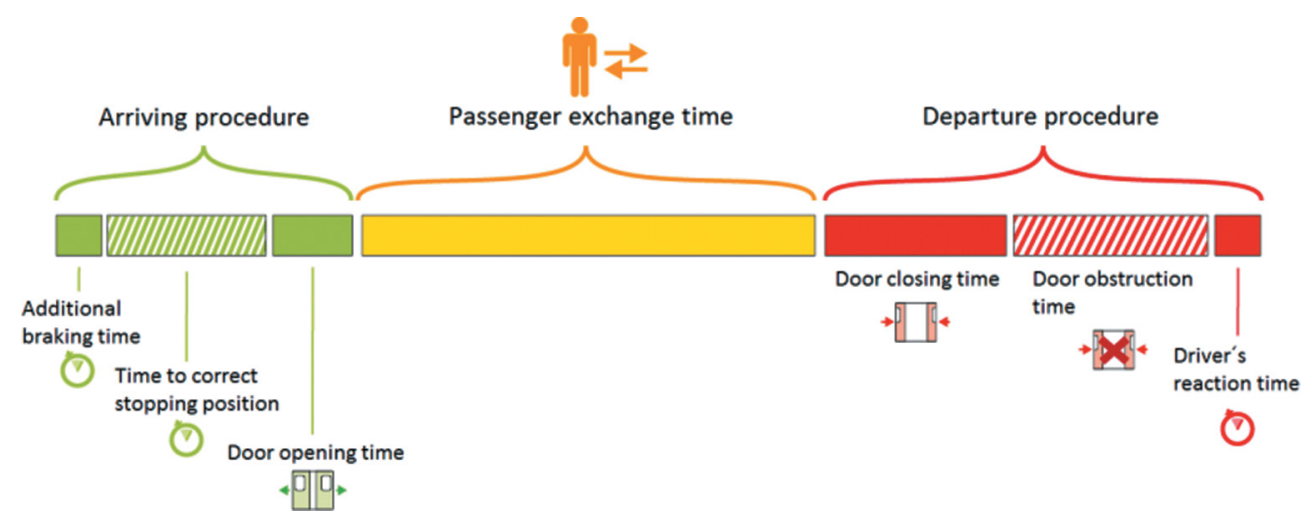

Figure 5: Factors causing dwell times in stations with platform screen doors. 
have been assumed to be the same as in existing stations. Thus, Stockholm City is considered to correspond to the current Stockholm Central station and Stockholm Odenplan is considered to correspond to Årstaberg/Stockholms Södra.

The door closing procedure will follow one of the three alternatives. The time for these procedures has been measured to be 16,17 and $15 \mathrm{~s}$, respectively, as discussed earlier.

From time to time a train departure is delayed due to passengers who obstruct the doors. Interviewed train conductors estimate that obstructions occur as often as in $60 \%$ of the stops at Stockholm Central during rush hours.

Two types of obstructions occur: short and long, depending on whether the obstruction is short enough to let the doors close successively automatically or the conductor has to repeat the closing procedure for the obstructed door. Measured values of dwell time extension due to obstruction and conductors' estimates of frequencies are shown in Table 1.

The last factor, driver's reaction time, is measured to be $4 \mathrm{~s}$ and corresponds to the time for the conductor to give 'clear to drive' announcement and the driver to react on this clearance message.

Four of the terms are assumed to be constants, whereas the other three are known to be stochastic with a high degree of variance: time to correct stopping position, passenger exchange time and door obstruction time. The Monte Carlo approach was used in order to convolute these three distributions to one total dwell time distribution for each of the two stations: Stockholm City and Stockholm Odenplan. The four constants were added to these distributions, resulting in the total distributions as shown in Figs. 6 and 7.

Table 1: Data for door obstructions.

\begin{tabular}{lccc}
\hline & \multirow{2}{*}{ Dwell time extension (s) } & \multicolumn{2}{c}{ Estimated frequency } \\
\cline { 3 - 4 } & & Stockholm Odenplan & Stockholm City \\
\hline Short obstruction & 5 & $17 \%$ & $50 \%$ \\
Long obstruction & 18 & $2 \%$ & $10 \%$ \\
\hline
\end{tabular}

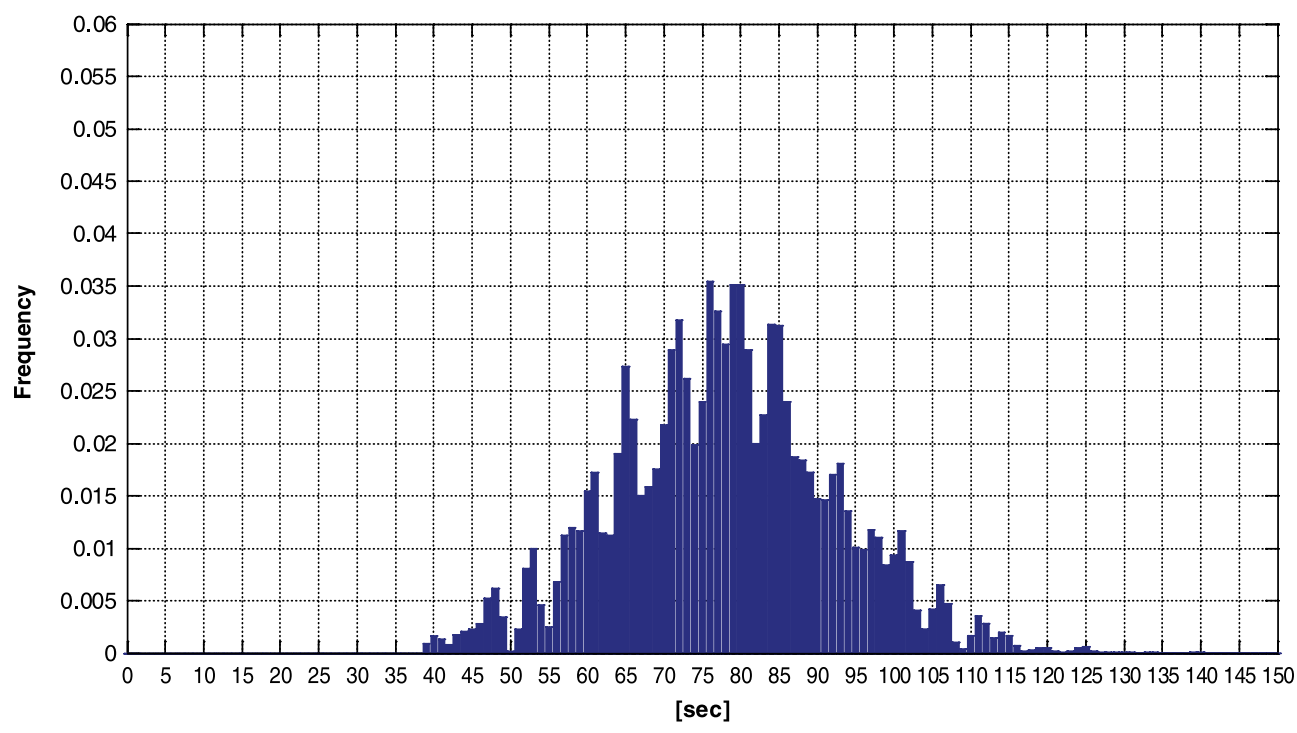

Figure 6: Dwell time distribution for Stockholm City. 


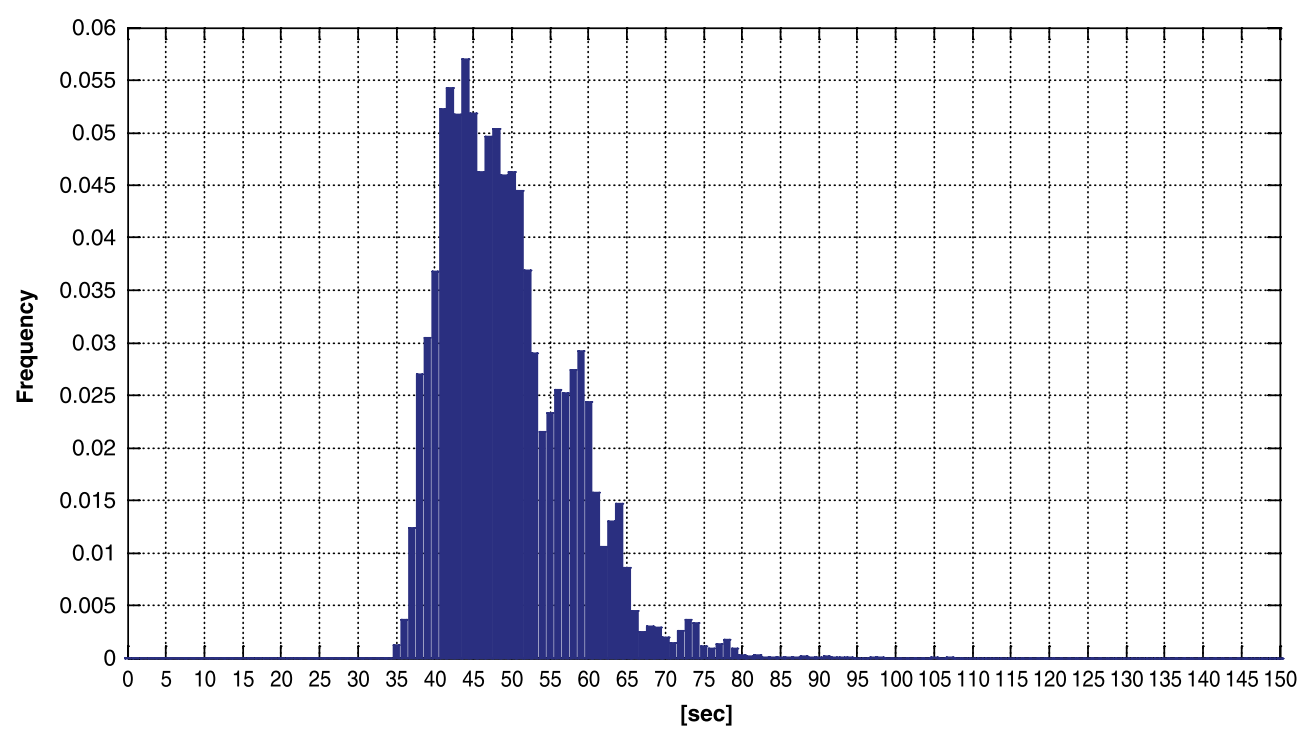

Figure 7: Dwell time distribution for Stockholm Odenplan.

The mean dwell time in Stockholm City is $78 \mathrm{~s}$ and in Stockholm Odenplan $50 \mathrm{~s}$. This fact gives some very interesting results: the dwell time in Stockholm Odenplan is about $12 \mathrm{~s}$ longer than that in Årstaberg and Stockholms Södra, although the time for passenger exchange is the same. This is the expected capacity cost for the platform screen doors. Since Stockholm Odenplan is a two-track station, it is reasonable to believe that this station will become the bottleneck of the line.

On the other hand, Stockholm City proves to be a station where trains will recover delays, since the scheduled dwell time, $180 \mathrm{~s}$, is well above even the longest dwells in the distribution.

\section{SIMULATION RESULTS}

A detailed infrastructure model for the system Älvsjö-Sundbyberg/Solna was built up in RailSys (Radtke [6]) and timetables for the five levels of capacity utilization were constructed. Each timetable included four traffic patterns, corresponding to the four branches in the network.

The four distributions for initial delays were assigned in Älvsjö for northbound traffic (two patterns) and in Sundbyberg and Solna for southbound traffic. Distributions for dwell times were assigned as follows:

- Årstaberg and Stockholms Södra: distribution for total dwell time compiled directly from measurements in Årstaberg station (see Fig. 4).

- Stockholm City and Stockholm Odenplan: distribution derived from Monte Carlo simulation (see Figs. 6 and 7).

These distributions for dwell time were adjusted in three levels to reflect the effect of the three alternative door closing procedures in Stockholm City and Stockholm Odenplan that were to be evaluated.

Figures 8 and 9 show the simulation results as mean departure delay for all stations. The mean arrival delays at Stockholm City are also included in the figures, since the scheduled 
dwell time in this station contains time supplements for delay recovery. Mean delays for the existing system are also shown for comparison.

Northbound traffic shows the expected effects of the traffic separation that is the main aim of the City line. The commuter trains can pass through Stockholm without interaction with regional and long-distance trains. This is shown in significantly lower delays. The useful capacity in this direction would be at least 24 trains/hour, given that all assumptions hold. Stockholm Odenplan is a bottleneck, which is reflected in increase of delay, whereas the longscheduled dwell time in Stockholm City gives a good recovery effect. It is a considerable advantage for northbound traffic that the recovery is located just before the most capacityrestrictive passage of Stockholm Odenplan.

Southbound traffic shows less useful capacity, if mean delay is to be decreased compared to the current system with mixed traffic. This is due to the fact that the bottleneck station, Stockholm Odenplan, is located before the station where trains recover delays.

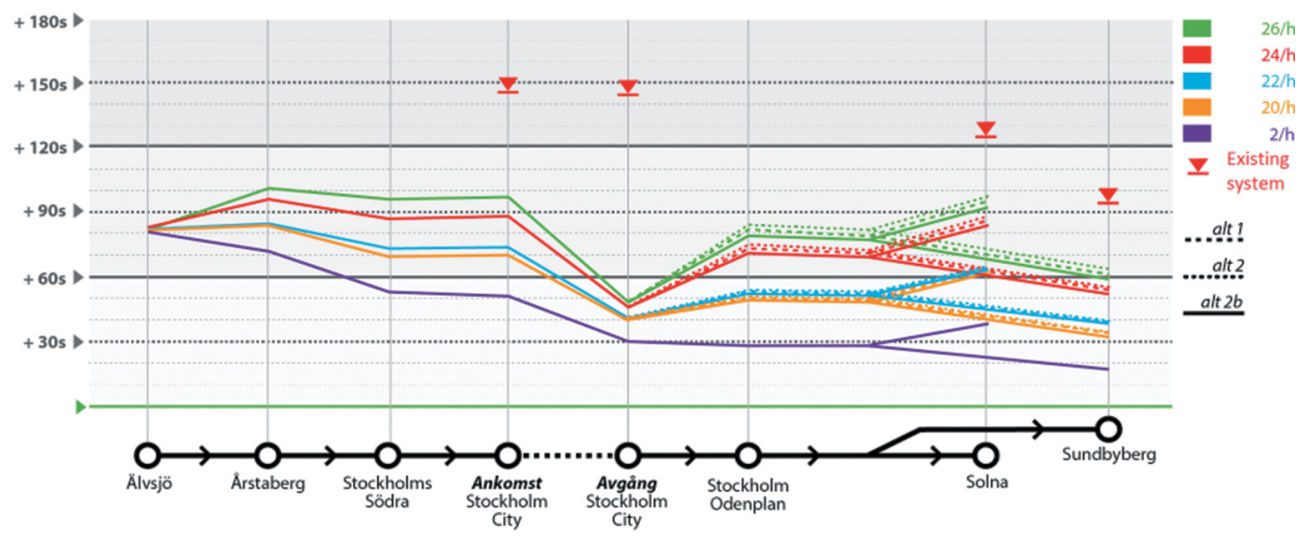

Figure 8: Simulation results for northbound traffic. The diagram indicates mean departure delays during rush hours for all stations. For Stockholm City both mean arrival and departure delays are presented.

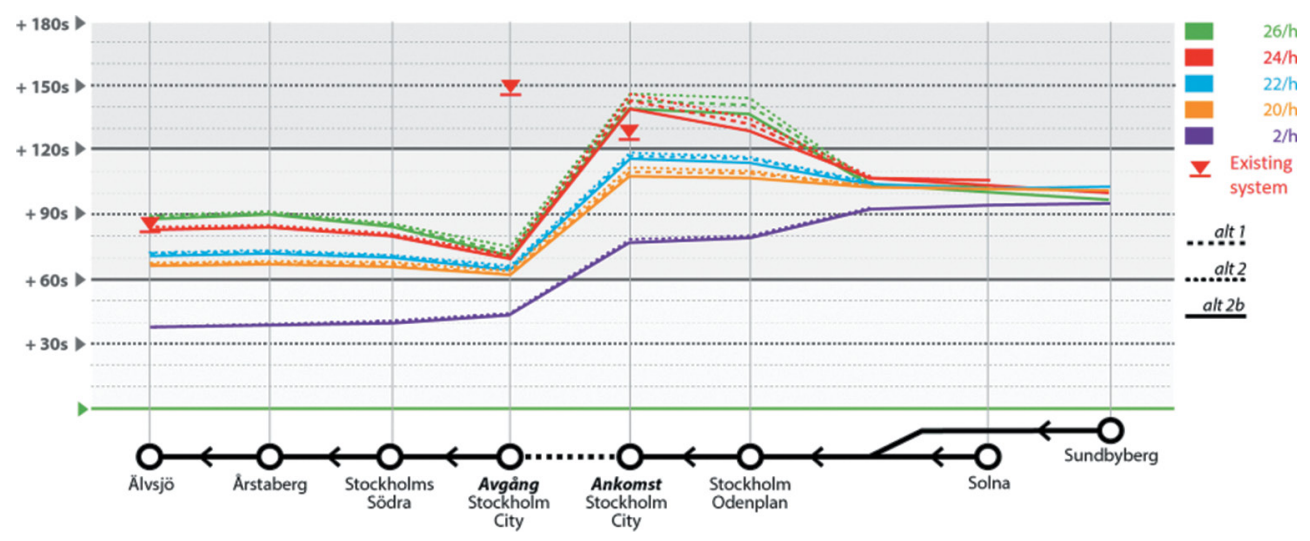

Figure 9: Simulation results for southbound traffic. The diagram indicates mean departure delays during rush hours. For Stockholm City both mean arrival and departure delays are presented. 
The variance in arrival delay in Stockholm Odenplan is rather high and it results in train queues and delay propagation. The useful capacity for southbound traffic is therefore only 22 trains/hour, if mean arrival delay in Stockholm City is not allowed to exceed the current arrival delay in Stockholm Central station.

The differences between alternative door closing procedures are relatively small, about five s/train as a maximum. The capacity utilization has generally a greater impact on mean delays.

\section{CONCLUSIONS}

This paper explores how simulation can be used to determine useful capacity for a commuter line and how the effect of platform screen doors on capacity can be estimated through simulation.

Since the platform screen doors extend dwell times, the effect on capacity can be modelled through adjustments of the dwell time distribution in stations equipped with platform screen doors. The compilation and derivation of these distributions constitute a core activity in this simulation study, whereas the simulation itself follows ordinary standards.

The study indicates that the platform screen doors reduce the useful capacity, since they extend the dwell time by $12 \mathrm{~s}$ (mean value). The main goal, to operate 24 trains/hour/direction on the City line, is difficult to achieve, especially in the southbound direction where the bottleneck station is located right before the four-track station where scheduled dwell time can be extended in order to allow trains to recover delays.

The useful capacity might be even lower, since no failure rates for neither the screen doors nor other parts of the infrastructure or vehicles have been taken into account in the simulation studies. A straight comparison to empirical delay data from the current system is therefore not easily performed.

\section{REFERENCES}

[1] Lindfeldt, O., Railway Operation Analysis. Evaluation of Quality, Infrastructure and Timetable on Single and Double-Track Lines with Analytical Models and Simulation. Royal Institute of Technology: Stockholm. Thesis (PhD), 2010.

[2] Sipilä, H., Simulation of Rail Traffic: Methods for Timetable Construction, Delay Modeling and Infrastructure Evaluation. Royal Institute of Technology: Stockholm. Thesis (PhD), 2015.

[3] Pachl, J., Timetable design principles. Railway Timetable and Traffic, eds. I.A. Hansen \& J. Pachl, Eurailpress: Hamburg, pp. 9-42, Ch. 2, 2008.

[4] Yuan, J. \& Hansen I.A., Optimizing capacity utilization of stations by estimating knock-on delays. Transportation Research Part B 41, 202-217, 2007. DOI: 10.1016/j. trb.2006.02.004.

[5] Swedish Transport Administration. Delay statistics for commuter trains in Stockholm 2013-01-01 - 2013-12-31.

[6] Radtke, A., Software Tools to Model the Railway Operation, Habilitation at the University of Hannover, Hannover, 2005. 